\title{
Pseudoaneurysm Formation in Intracerebral Hematoma due to Ruptured Middle Cerebral Artery Aneurysm
}

\author{
Motohiro Nomura ${ }^{1}$ Akira Tamase ${ }^{1}$ Tomoya Kamide $^{1}$ Kentaro Mori $^{1}$ Syunsuke Seki ${ }^{1}$ \\ Kunio Yanagimoto ${ }^{2}$
}

${ }^{1}$ Department of Neurosurgery, Yokohama Sakae Kyosai Hospital,

Address for correspondence Motohiro Nomura, MD, PhD, Sakae-ku, Yokohama, Japan

2 Department of Pathology, Yokohama Sakae Kyosai Hospital, Sakae-ku, Yokohama, Japan Department of Neurosurgery, Yokohama Sakae Kyosai Hospital, 132 Katsura-cho, Sakae-ku, Yokohama 247-8581, Japan (e-mail: nomura413jp@yahoo.co.jp).

Surg J 2015;1:e47-e49.

\begin{abstract}
We report the case of a ruptured middle cerebral artery aneurysm that showed pseudoaneurysm formation in an intracerebral hematoma. A 61-year-old man who was taking warfarin complained of dysarthria. Three days later, he was found unconscious, and computed tomography on admission showed subarachnoid hemorrhage and an intracere-

Keywords

- pseudoaneurysm

- cerebral aneurysm

- subarachnoid hemorrhage

- intracerebral hematoma bral hematoma in the left temporal lobe. Three-dimensional computed tomographic angiography showed an irregular-shaped aneurysm-like cavity extending from the left middle cerebral artery into the hematoma. Intraoperative observation revealed that the aneurysm itself was small and the lesion observed on computed tomography was a pseudoaneurysm that had formed in the hematoma. Pathologic examination of the aneurysm demonstrated that there was a thrombus at its tip. In this report, radiologic characteristics of three-dimensional computed tomographic angiography and etiology of a pseudoaneurysm in an intracerebral hematoma due to aneurysm rupture are discussed.
\end{abstract}

Pseudoaneurysms usually result from trauma, mycotic infection, vessel dissection, or congenital collagen deficiency. ${ }^{1-3}$ We previously reported the case of a pseudoaneurysm in a thrombus located at the rupture site of a cerebral aneurysm. ${ }^{2}$ Since then, a few reports describing pseudoaneurysm formation after aneurysmal rupture have been published. ${ }^{4-6}$ However, this condition is still extremely rare and remains to be clarified. We encountered the case of a large pseudoaneurysm that had formed in an intracerebral hematoma due to the rupture of a saccular aneurysm of the middle cerebral artery (MCA). We initially diagnosed this case as a large MCA aneurysm presenting with subarachnoid hemorrhage (SAH) and an intracerebral hematoma based on the findings of three-dimensional computed tomographic angiography (3D-CTA). However, intraoperative observation revealed a medium-sized aneurysm of the MCA. In this report, we describe the radiologic findings on 3D-CTA and etiology of a pseudoaneurysm formed in an intracerebral hematoma due to a ruptured cerebral aneurysm.

received

June 23, 2015

accepted

September 14, 2015

published online

November 19, 2015

\section{Case Report}

A 61-year-old man initially developed speech disturbance. This patient had a history of aortic valve replacement due to aortic regurgitation and was taking warfarin. Two days later, his son recognized that he showed motor aphasia. Three days after the initial symptom, he was found unconscious at home and brought to our hospital by ambulance. Computed tomography (CT) showed SAH and an intracerebral hematoma in the left temporal lobe (-Fig. 1a). Contrast-enhanced CT revealed an enhanced lesion in the hematoma ( - Fig. 1b). 3D-CTA showed a cavity extending from the MCA into the hematoma ( - Fig. 1c). We initially suspected SAH and an intracerebral hematoma due to rupture of a large MCA aneurysm. The intravenous administration of menatetrenone and fresh frozen plasma was conducted immediately after CT. Angiography was not performed, because the $\mathrm{CT}$ showed brain herniation and there was insufficient time for angiography. Following $\mathrm{CT}$, emergency surgery

Copyright $\odot 2015$ by Thieme Medical Publishers, Inc., 333 Seventh Avenue, New York, NY 10001, USA. Tel: +1(212) 584-4662.
License terms

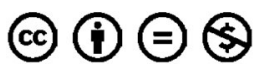

$10.1055 / \mathrm{s}-0035-1567877$ ISSN 2378-5128. 

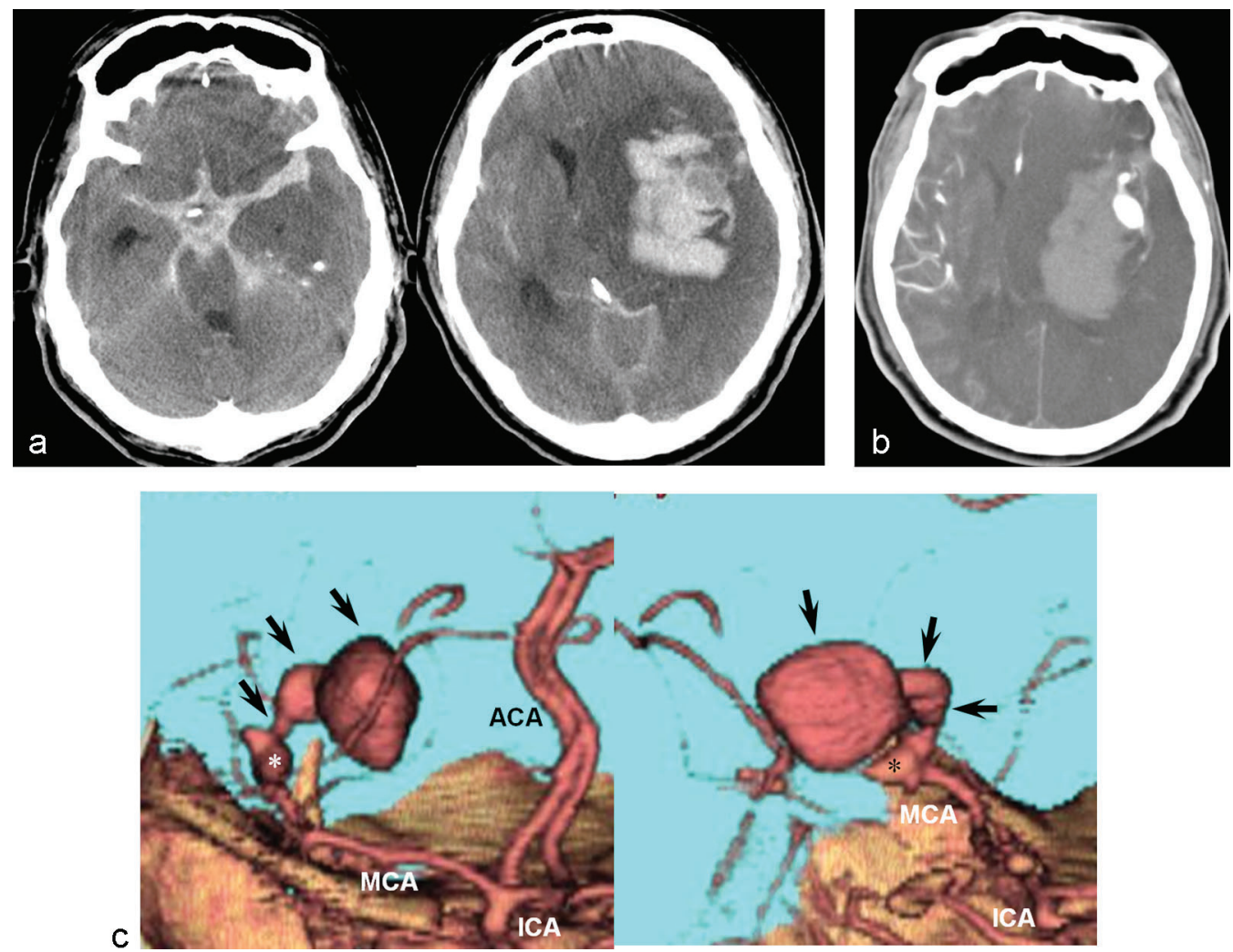

Fig. 1 (a) Computed tomography (CT) showing subarachnoid hemorrhage and a hematoma in the left temporal lobe. (b) Contrast-enhanced CT showing an enhanced tortuous lesion in the hematoma. (c) Three-dimensional computed tomographic angiography showing a large aneurysmlike cavity (arrows). Asterisk indicating the aneurysm. Abbreviations: ACA, anterior cerebral artery; ICA, internal carotid artery; MCA, middle cerebral artery.

was performed. Upon dural incision, the brain showed swelling due to increased intracranial pressure. Therefore, prior to exposure of the aneurysm, the intracerebral hematoma was partially removed. The hematoma was relatively hard and could not be suctioned, suggesting that it was not fresh. Intraoperative findings showed that the M1 terminal of the MCA was swollen and divided into three M2 branches, and there was a medium-sized aneurysm protruding into the temporal side at the MCA trifurcation (-Fig. 2a). The aneurysm neck was initially exposed and clipped (-Fig. 2b). Then, the aneurysm dome was dissected. No large aneurysm cavity as demonstrated on 3D-CTA was found. The tip of the aneurysm dome was incised and subjected to pathologic examination. The bone flap was not returned, because the intracranial pressure was very high.

Pathologic examinations revealed destruction of the aneurysm wall with the significant infiltration of eosinophils, indicating that a few days had passed since the aneurysm rupture (-Fig. 2 c).

Postoperatively, he was treated with mannitol to lower the brain tension. However, he passed away 5 days after the operation.

\section{Discussion}

Pseudoaneurysm formation at the rupture site of a cerebral aneurysm is very rare. ${ }^{2,5} \mathrm{~A}$ pseudoaneurysm is formed in a blood clot adhering to the rupture point. We encountered the case of a pseudoaneurysm that had formed in a massive intracerebral hematoma, not in a small blood clot at the rupture point. To our knowledge, this is the first reported case of a pseudoaneurysm in an intracerebral hematoma.

Mori et al reported two cases of pseudoaneurysms that had formed in a thick SAH. ${ }^{6}$ They reported that pseudoaneurysms showed the peculiar findings of a delayed appearance, changing shape, retention of contrast medium after the venous phase, and an unclear neck location on angiography and 3D-CTA. They concluded that these radiologic findings of the aneurysms led to misdiagnoses as large partially thrombosed aneurysms. In our case, 3D-CTA showed a cavity extending from the MCA. On axial contrast-enhanced CT, a tortuous enhancing portion like a drainer of arteriovenous malformation was seen in the hematoma. The lesion appeared to have a thrombus in it. Based on the CT findings, we diagnosed the lesion as a large cerebral aneurysm originating from the MCA preoperatively.

Intraoperatively, the aneurysm was seen originating from the trifurcation of the MCA and buried in the hematoma of the temporal lobe. After neck clipping, the aneurysm was dissected and fully exposed. However, not a large but a medium-sized aneurysm was found. Intraoperative findings indicated that the hematoma was hard compared with common hematomas. This observation indicated that the hematoma was relatively old. In fact, this patient, who was on warfarin, developed the initial symptom 3 days prior to consciousness loss. Therefore, the 

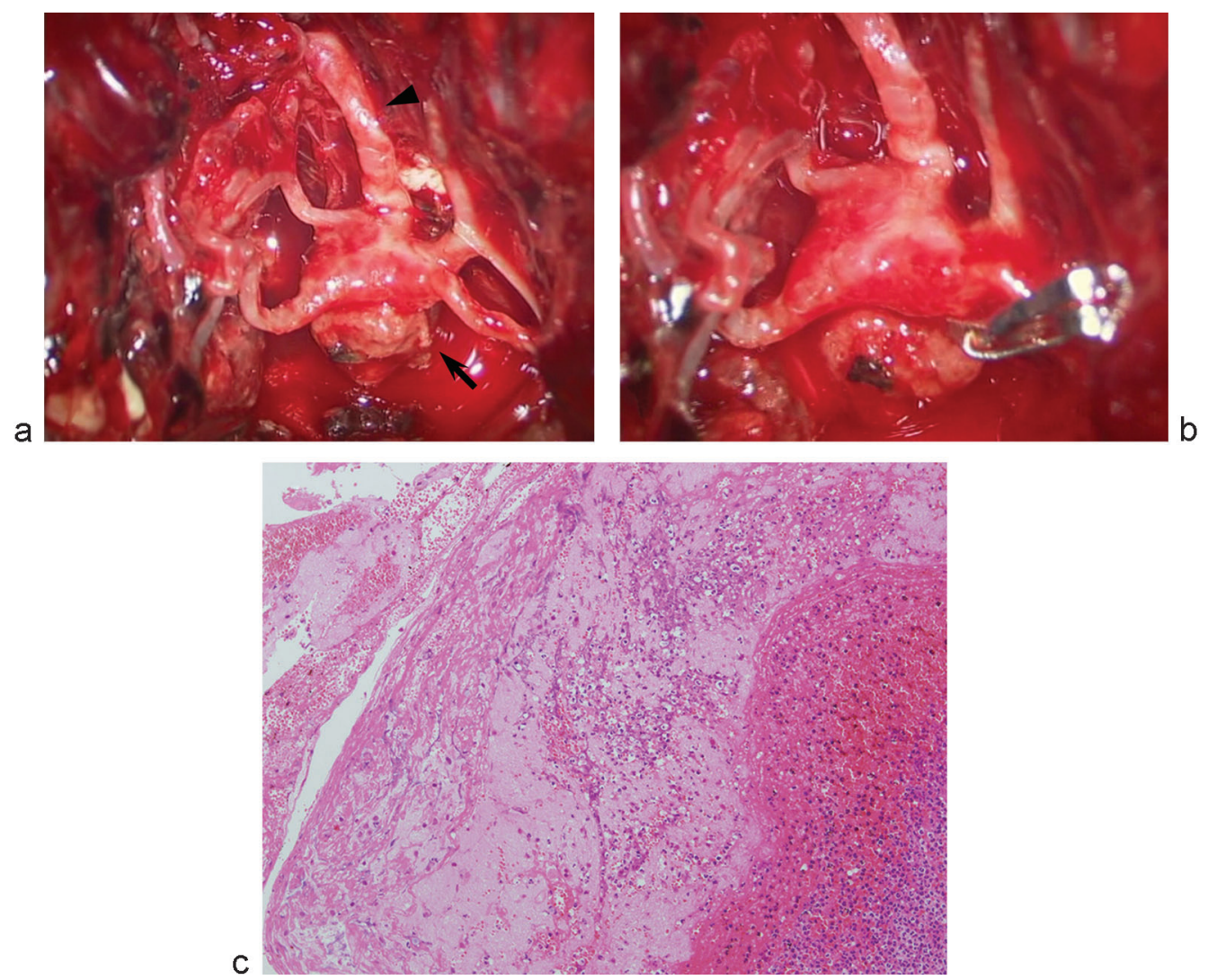

Fig. 2 (a) Intraoperative photograph showing an aneurysm (arrow) originating from the middle cerebral artery (arrowhead). (b) The aneurysm neck is clipped. (c) Microphotograph of the resected specimen demonstrating a thrombus and destroyed aneurysm wall. Eosinophils are infiltrating the aneurysm wall. (Hematoxylin and eosin, $\times 100$.)

aneurysm might have ruptured 3 days before admission, resulting in minor bleeding. Then, massive hemorrhage extending into the temporal lobe from the aneurysm occurred. After that, additional bleeding from the aneurysm may have occurred and formed a pseudoaneurysm in the hematoma under the influence of inhibited coagulation due to warfarin. Mori et al speculated that the initial bleeding from the aneurysm formed a large hematoma on the dome of the aneurysm, and the second rupture formed a communicating lumen in the hematoma in their cases. ${ }^{6}$

Pathologic examination revealed that the lesion was composed of a destroyed aneurysm wall and red thrombus. The destroyed aneurysm wall showed infiltration by eosinophils, indicating that some time had passed since rupture of the aneurysm.

Based on intraoperative and pathologic findings, the aneurysm was medium-sized. Also, a pseudoaneurysm, a cavity continuing from the aneurysm, had formed in the intracerebral hematoma. The pseudoaneurysm cavity was opacified like a large aneurysm of the MCA.

\section{Conclusion}

Pseudoaneurysm formation in an intracerebral hematoma is extremely rare. However, in cases of an irregular-shaped aneurysm cavity accompanying an intracerebral hematoma, a pseudoaneurysm should be taken into consideration as a differential diagnosis.

\section{References}

1 Lempert TE, Halbach VV, Higashida RT, et al. Endovascular treatment of pseudoaneurysms with electrolytically detachable coils. AJNR Am J Neuroradiol 1998;19(5):907-911

2 Nomura M, Kida S, Uchiyama N, et al. Ruptured irregularly shaped aneurysms: pseudoaneurysm formation in a thrombus located at the rupture site. J Neurosurg 2000;93(6):998-1002

3 Teitelbaum GP, Dowd CF, Larsen DW, et al. Endovascular management of biopsy-related posterior inferior cerebellar artery pseudoaneurysm. Surg Neurol 1995;43(4):357-359

4 D'Urso PI, Loumiotis I, Milligan BD, Cloft H, Lanzino G. "Real time" angiographic evidence of "pseudoaneurysm" formation after aneurysm rebleeding. Neurocrit Care 2011;14(3):459-462

5 Ide M, Kobayashi T, Tamano Y, Hagiwara S, Tanaka N, Kawamura H. Pseudoaneurysm formation at the rupture site of a middle cerebral artery aneurysm-case report. Neurol Med Chir (Tokyo) 2003; 43(9):443-446

6 Mori K, Kasuga C, Nakao Y, Yamamoto T, Maeda M. Intracranial pseudoaneurysm due to rupture of a saccular aneurysm mimicking a large partially thrombosed aneurysm ("ghost aneurysm"): radiological findings and therapeutic implications in two cases. Neurosurg Rev 2004;27(4):289-293 